\title{
Síntomas psicopatológicos y dimensiones de personalidad en adolescentes con discapacidad visual: cambios evolutivos y relaciones entre ambas variables
}

\author{
Maite Garaigordobil y Elena Bernarás \\ Universidad del País Vasco \\ Correspondencia: Maite Garaigordobil. Departamento de Personalidad, Evaluación y \\ Tratamiento Psicológicos. Facultad de Psicología. \\ Universidad del País Vasco. Avda. de Tolosa, 70 - 20018 Donostia-San Sebastián. \\ Correo electrónico: maite.garaigordobil@ehu.es
}

\section{RESUMEN}

El estudio tuvo 3 objetivos: 1) analizar diferencias entre sexos y cambios con la edad en síntomas psicopatológicos y dimensiones de personalidad en adolescentes con discapacidad visual (DV); 2) valorar diferencias entre los adolescentes con y sin DV en psicopatología y personalidad; y 3) explorar las relaciones entre psicopatología y personalidad. La muestra contiene 29 participantes con DV de 12 a 20 años. Se administraron el SCL-90-R y NEO-FFI. Los resultados confirmaron que las chicas con DV tuvieron puntuaciones superiores a los chicos con DV en varios síntomas psicopatológicos. La comparación entre adolescentes con y sin DV evidenció que las chicas con DV tenían niveles altos de psicopatología, mientras que los chicos con DV tenían niveles medios; y ambos sexos mostraron baja extraversión. Los síntomas no aumentaron con la edad, y la responsabilidad disminuyó. Se encontraron correlaciones positivas entre neuroticismo y síntomas psicopatológicos; y negativas de extraversión y amabilidad con algunos síntomas.

Palabras Clave: discapacidad visual, psicopatología, personalidad, adolescencia, género.

\section{ABSTRACT}

The purpose of this study is three-fold: 1) to determine whether there are sex differences in the changes that occur with age in psychopathological symptoms and personality dimensions in adolescents with visual impairment (VI);2) to appraise whether there are differences among the adolescents with and without VI in psychopathology and personality; and 3) to explore the relations between psychopathology and personality. The sample comprised 29 participants with $\mathrm{VI}$, aged from 12 to 20 years. The SCL-90-R and the NEO-FFI were administered. The results showed that girls with VI obtained higher scores than boys with VI in various psychopathological symptoms. The comparison between adolescents of both sexes with and without VI showed that the girls with VI had higher scores in general psychopathology, whereas the boys with VI had medium levels; and both had low extraversion. The symptoms did not increase with age, and the responsibility decreased. Positive correlations were found between neuroticism and symptoms of psychopathology, and negative associations of extraversion and kindness to some symptoms.

Keywords: visual impairment, psychopathology, personality, adolescence, gender. 


\section{Introducción}

La revisión de los estudios sobre síntomas psicopatológicos y dimensiones de personalidad realizados con personas con DV pone de relieve que la mayor parte de éstos se han realizado con adultos y ancianos, siendo escasos los trabajos que han explorado estas variables en niños y adolescentes con esta discapacidad. El trabajo de Ammerman, Van Hasselt y Hersen (1986), que examinó los aspectos psicológicos, psiquiátricos, y la literatura de la visión en 4 áreas de ajuste (funcionamiento intelectual, características de la personalidad, desarrollo social, y psicopatología) en los estudios llevados a cabo con niños y adolescentes con discapacidad visual (DV), concluye que existe un número importante de variables intervinientes (etiología de la pérdida de la visión, el grado de deterioro ) que influyen en el desarrollo de los discapacitados visuales. No obstante, sugieren que aunque la DV en niños y adolescentes puede dar lugar a una situación de alto riesgo de disfunción psicológica, esta discapacidad no es en sí misma necesariamente una causa de mal ajuste. En esta dirección ya habían apuntado estudios previos, que habiendo demostrado alguna relación entre sintomatología neurótica y discapacidad, concluyeron que los problemas de conducta en niños y adolescentes ciegos provienen sobre todo de factores externos como el ambiente familiar, la institucionalización, la reacción de las personas videntes hacia los ciegos... (Freedman, 1967; Norris, Spaulding y Brodie, 1957).

En la década de los 70, en un estudio realizado en Grecia (Dimitriou, 1973) con una muestra de 100 estudiantes de una escuela para ciegos (59 varones y 41 mujeres, edad media de los varones 17,3 años y 19,0 en las mujeres), algunos de ellos manifestaron características neuróticas. Concretamente, 27 estudiantes (11 varones y 16 mujeres) habían desarrollado un completo cuadro clínico neurótico. El más joven de los estudiantes tenía 9 años y el mayor 30. Los síntomas que presentaban eran excesiva ansiedad, palpitaciones, sudoración en las manos, temblor, insomnio, dolores de cabeza... Las mujeres mostraban el doble de neuroticismo que los varones (39\%-18.6\%).

En relación a síntomas psicopatológicos asociados a la DV, Huurre, y Aro (1998) realizaron un estudio con 54 adolescentes con DV (edad media 14 años) y 385 adolescentes con visión normal del mismo nivel de edad, cuyos resultados evidenciaron que el grupo de adolescentes con DV no diferían de los de control en la frecuencia de la depresión y de los síntomas de angustia. Sin embargo, el estudio exploratorio de Koenes y Karshmer (2000) realizado con una muestra de 22 adolescentes ciegos desde el nacimiento y 29 adolescentes sin problemas visuales confirmó que la incidencia de la depresión en los adolescentes ciegos fue significativamente mayor en relación a los videntes. El estudio de Garaigordobil y Bernarás (2009) también confirmó que los adolescentes con DV frente a los de control con visión normal tenían significativamente más síntomas psicopatológicos (obsesión-compulsión, hostilidad, ideación paranoide, adicionales) y un mayor nivel global de psicopatología (GSI), así como de distrés por los síntomas (PSDI); sin embargo, no se encontraron diferencias en depresión al comparar los adolescentes con DV 
con 61 adolescentes sin DV. Trabajos llevados a cabo con adultos con DV (edad media 60 años) (Noran, Izzuna, Bulgiba, Mimiwati y Ayu, 2009) muestran relaciones positivas entre la gravedad de la discapacidad y el nivel de depresión. Y en la misma dirección, los análisis de regresión logística realizados en el estudio de Chou y Chi (2004) con adultos de 60 o más años de edad revelaron que la DV estaba relacionada con depresión incluso con mayor fuerza que la deficiencia auditiva.

Otro grupo de investigaciones ha puesto de relieve las dificultades de socialización de los adolescentes con DV. Burlingan (1979) encontró que los jóvenes con DV tenían algunas dificultades en su comportamiento social (mayor dependencia de los demás, falta de iniciativa, menos agresividad o más ansiedad). Huurre y Aro (1998) estudiando el desarrollo psicosocial de 54 adolescentes con DV (edad media 14 años) con el contraste de un grupo de control de 385 adolescentes con visión normal del mismo nivel de edad, evidenciaron que los adolescentes con DV tenían menos amigos y citas con otros jóvenes que los que no tenían discapacidad. También tenían más sentimientos de soledad y dificultades para hacer amigos. La autoestima, el rendimiento escolar y habilidades sociales fueron menores en las niñas con DV que en las de control. En resumen, los resultados mostraron que algunos adolescentes con DV, especialmente las niñas, necesitan más apoyo en su desarrollo psicosocial. Posteriormente, Huurre y Aro (2000) comparan el bienestar psicosocial de 115 adolescentes finlandeses con problemas visuales (distintos grados de DV) con 44 adolescentes con enfer- medades crónicas y con 607 adolescentes sin discapacidades ni problemas. Este estudio realizado con adolescentes de 12 a 17 años concluyó que los adolescentes con DV, especialmente aquellos que eran ciegos, tuvieron más dificultades en sus relaciones con los amigos que los de los otros dos grupos, pero mostraron menos problemas con el bienestar psicológico que los adolescentes con enfermedades crónicas. También, Fiesler, Lechner, y Bos (2008) concluyen que la ceguera está relacionada con mayor frecuencia de sentimientos de miedo, $y$ menor apoyo social. En relación a dimensiones de personalidad, el estudio realizado con personas de 60 años (Casten, Rovner, y Edmonds, 2001) no encontró relaciones entre medidas de personalidad (neuroticismo, apertura, amabilidad, extraversión) y DV.

Apenas existen estudios que hayan analizado las correlaciones entre síntomas psicopatológicos y dimensiones de personalidad durante la infancia y la adolescencia en adolescentes con DV. En este sentido cabe resaltar el estudio del Instituto de Tecnología de la India (Satapathy y Singhal, 2001) que analizó con una muestra de 79 adolescentes con DV de 13 a 21 años, las relaciones entre ajuste socioemocional y diversas variables de personalidad. Los resultados revelaron que el ajuste socioemocional correlacionaba significativamente de forma negativa con el estrés, con problemas conductuales, con conductas de retraimiento y con falta de atención, observándose correlaciones positivas con autoestima. Aunque, con muestras adultas, Rovner y Casten (2001) estudiaron las conexiones entre la degeneración macular que se produce en la edad y depresión en una 
muestra de 51 pacientes ancianos (edad media 79 años), confirmando que el rasgo de la personalidad neuroticismo fue altamente predictivo de la depresión.

La adolescencia es una etapa de la vida compleja en la que se inician cambios físicos, psicológicos y sociales que proporcionan a los jóvenes las capacidades de las personas maduras. Si a esta difícil situación de cambio e inestabilidad propia de la etapa, se le añade una DV, esta etapa de la vida se complica aún más. En el ámbito de la DV no son muchos los estudios que se realizan con población escolarizada en torno a aspectos tan importantes como son los síntomas psicopatológicos y rasgos de personalidad. Tanto los padres como el profesorado de este alumnado siente necesidad de conocer en qué ámbitos o aspectos pueden necesitar ayuda y este estudio pretende profundizar en esta dirección.

Con esta contextualización, el presente estudio realizado con adolescentes con DV tuvo 3 objetivos: 1) analizar si existen diferencias entre sexos y cambios con la edad en diversos síntomas psicopatológicos (somatización, obsesión-compulsión, ansiedad, depresión, sensibilidad interpersonal, ansiedad fóbica, ideación paranoide, psicoticismo) y en dimensiones de personalidad (neuroticismo, extraversión, apertura, amabilidad, responsabilidad); 2) valorar si existen diferencias entre los adolescentes con y sin DV de ambos sexos en psicopatología y personalidad, utilizando para esta comparación las muestras de tipificación de los tests utilizados; y 3 ) explorar las relaciones existentes entre síntomas psicopatológicos y dimensiones de personalidad.
En la investigación se proponen 4 hipótesis: 1) las chicas con DV tendrán significativamente más síntomas psicopatológicos que los chicos con discapacidad, sin embargo, no se encontrarán diferencias en las dimensiones de personalidad; 2) los y las adolescentes con DV comparados con los que tienen visión normal tendrán puntuaciones superiores en el índice global de psicopatología y en el rasgo de personalidad neuroticismo; 3) dadas las dificultades asociadas a la situación de DV se postula que a medida que aumenta la edad habrá un incremento de los síntomas psicopatológicos y del neuroticismo como rasgo de personalidad, mientras que en extraversión, apertura, amabilidad y responsabilidad no habrá variaciones significativas con la edad; además, el sexo mediará los cambios evolutivos observándose que las chicas tendrán un mayor nivel de cambio en esta dirección; y 4) se encontrarán relaciones positivas entre síntomas psicopatológicos y la dimensión de personalidad neuroticismo, así como correlaciones negativas con extraversión, amabilidad y responsabilidad.

\section{Método \\ Participantes}

La muestra está configurada con 29 participantes con DV grave de 12 a 20 años de edad, pertenecientes a las tres provincias de la Comunidad Autónoma del País Vasco (CAPV). La muestra fue seleccionada del censo de adolescentes escolarizados en centros de primaria y secundaria $(n=60)$. La muestra fue identificada a través de los Centros de Recursos para la Inclusión educativa del alumnado con DV (CRI) situados en las tres capitales de la Comu- 
nidad Autónoma Vasca. Los profesores de estos centros colaboraron en el intercambio de documentación con las familias y facilitaron el acceso al alumnado. En primer lugar se identificaron los 60 adolescentes escolarizados en la CAPV con DV, con edades comprendidas entre 12 y 20 años, se eliminaron aquellos que presentaban otras discapacidades asociadas, y al resto $(n=47)$ se les envió una carta informando del objetivo de la investigación y solicitando su participación. De ellos, 29 dieron su consentimiento informado participando en el estudio. Todos los participantes estaban estudiando en centros educativos, públicos, privados y concertados. En cuanto al sexo, $16(55,2 \%)$ son varones y 13 (44,8\%) mujeres. En el momento de la investigación 23 cursaban Educación Primaria (79,3\%), y 6 Secundaria (20,6\%). Entre las características que presenta la muestra, en cuanto al grado de visión en función de la pérdida de agudeza, un 24,1\% tiene un nivel de visión entre el 0,4 y el 0,3\%; el 27,6\% entre el 0,25 y 0,12 ; el 34,5\% tiene una visión igual o inferior a 0,1 , y el 6,9\% tiene un nivel de deterioro visual total o amaurosis. En cuanto a la pérdida de campo visual el $10,3 \%$ no supera los $40^{\circ}$ de visión respecto a los $180^{\circ}$ usuales y el 10,3\% no supera los $20^{\circ}$. Entre las patologías identificadas: el 6,9\% tiene ambliopía (ojo vago), el 34,5\% miopía, el 3,4\% astigmatismo; el 6,9\% miopía y astigmatismo, el 10,3\% estrabismo, el 37,9\% nistagmus, el 13,8\% malformaciones oculares, el 10,3\% alteraciones congénitas oculares, el 3,4\% glaucoma, el 6,9\% cataratas, y el $34,4 \%$ lesiones en la retina. Un $82,8 \%$ de los sujetos sufre la pérdida visual desde el nacimiento, y el 17,2 \% restante experimentaron la pérdida entre el primer y el séptimo año de vida. En cuanto a la evolución de la patología visual, un $17,2 \%$ dice haber experimentado un empeoramiento, mientras que un 6,9\% dice haber mejorado su visión. El 55,2\% ha recibido atención temprana para su discapacidad, el $82,8 \%$ utiliza ayudas ópticas, el 79,3\% lee y escribe textos en tinta, mientras que el 10,3\% lee y escribe en braille, y el 10,3\% emplea ambos sistemas de lectura y escritura. El 31\% ha recibido entrenamiento visual, con el 10,3\% se han trabajado el tacto y el oído, y el 31\% han recibido entrenamiento para todos los sentidos. El $89,7 \%$ utiliza baja visión, el 79,3\% emplea nuevas tecnologías para el aprendizaje, y el 44,8\% ha participado en programas de orientación y movilidad. El 48,3\% está afiliado a la ONCE. Actualmente, únicamente uno de los participantes $(3,4 \%)$ acude al psicólogo debido a una depresión leve.

\section{Diseño y Procedimiento}

El estudio empleó una metodología descriptiva, comparativa y correlacional de corte transversal. Los adolescentes participaron voluntariamente $y$, previo consentimiento de sus padres, firmaron un protocolo de consentimiento informado, que previamente había sido autorizado por los jefes territoriales de Educación de cada Territorio Histórico. Las direcciones de los Centros de Recursos (CRI) facilitaron los nombres del alumnado que respondía a las características de la muestra que se había seleccionado a priori (sin otras patologías asociadas...). Los protocolos de consentimiento fueron enviados a cada CRI que a su vez los remitieron a las familias para 
su firma. De 47 alumnos a los que se envío el protocolo solamente respondieron 29 , que fueron finalmente los que formaron la muestra. La mayor dificultad fue la dispersión de la muestra a lo largo de las tres provincias de la CAPV, que suponía se necesitara prácticamente un día entero (horario escolar) para recoger 2 o 3 cuestionarios. Para medir la psicopatología y la personalidad se administraron de forma individual 2 instrumentos de evaluación en una sesión. La aplicación fue realizada por una psicóloga especialista en DV y 2 becarias de investigación formadas con esta finalidad.

\section{Instrumentos}

Con la finalidad de medir las variables dependientes se administran 2 instrumentos de evaluación con adecuadas garantías psicométricas de fiabilidad y validez.

SCL-90-R. Cuestionario de 90 síntomas revisado (Derogatis, 1983, adaptación española González de Rivera, De las Cuevas, Rodríguez Abuín, y Rodríguez Pulido, 2002). Este autoinforme está compuesto por 90 ítems distribuidos en 10 escalas psicopatológicas: somatización (vivencias de disfunción corporal, con alteraciones neurovegetativas de los sistemas cardiovascular, respiratorio, gastrointestinal y muscular), obsesión-compulsión (conductas, pensamientos e impulsos que el sujeto considera absurdos e indeseados, que generan intensa angustia y que son difíciles de resistir, evitar o eliminar), sensibilidad interpersonal (sentimientos de timidez y vergüenza, tendencia a sentirse inferior a los demás, hipersensibilidad a las opiniones $y$ actitudes ajenas $y$, en general, incomodidad e inhibición en las relaciones interpersonales), depresión (vivencias disfóricas, anhedonia, desesperanza, impotencia y falta de energía, ideas autodestructivas y otras manifestaciones cognitivas y somáticas características de los estados depresivos), ansiedad (manifestaciones clínicas de la ansiedad, tanto generalizada como aguda o "pánico", incluye signos generales de tensión emocional y sus manifestaciones psicosomáticas), hostilidad (pensamientos, sentimientos y conductas propios de estados de agresividad, ira, irritabilidad, rabia y resentimiento), ansiedad fóbica (miedo persistente, irracional y desproporcionado a un animal o persona, lugar, objeto o situación, generalmente complicado por conductas de evitación o de huida, con un mayor peso en la escala de los síntomas de agorafobia y fobia social que los de la fobia simple), ideación paranoide (conducta paranoide, que incluye suspicacia, centralismo autorreferencial e ideación delirante, hostilidad, grandiosidad, miedo a la pérdida de autonomía y necesidad de control), psicoticismo (espectro psicótico que se extiende desde la esquizoidia leve hasta la psicosis florida, y que en la población general se relaciona más con sentimientos de alienación social que con psicosis clínicamente manifiesta), y escala adicional (síntomas misceláneos que constituyen un claro referente de depresión melancólica). Además la prueba permite calcular el índice sintomático general (GSI), medida generalizada e indiscriminada de la intensidad del sufrimiento psíquico y psicosomático global, el total de síntomas positivos (PST), número de síntomas presentes, y el índice de distrés de síntomas positivos (PSDI), que relaciona el sufrimiento con los síntomas. Resultados de 
estudios con muestra española (González de Rivera et al., 2002) sugieren buena fiabilidad de la prueba, siendo coherentes con los del autor. Los coeficientes alpha oscilan entre 0,81 y 0,90. La estabilidad temporal (entre 0,78 y 0,90 ) con un intervalo test-retest de una semana muestra estabilidad de las puntuaciones a lo largo del tiempo. Estudios de validez muestran la relación entre el perfil de las dimensiones sintomáticas y el grupo diagnóstico al que pertenece la muestra clínica, encontrando puntuaciones significativamente mayores en las muestras clínicas que en las no clínicas (De las Cuevas y González de Rivera, 1991). Los estudios originales del autor con muestras americanas evidencian la validez de constructo (Derogatis y Cleary, 1977) y la convergente dadas las altas correlaciones con el MMPI en pacientes psiquiátricos (Derogatis, Rickels, y Rock, 1976).

NEO-FFI. Inventario de personalidad NEO reducido de cinco factores (Costa y MacCrae, 1999). El cuestionario consta de 5 escalas, de 12 ítems cada una, que permiten medir 5 grandes factores de personalidad: 1) Neuroticismo (puntuación alta: desajuste, inestabilidad emocional, tendencia general a experimentar sentimientos negativos, como miedo, melancolía, vergüenza, ira, culpabilidad y repugnancia; puntuación baja: ajuste, estabilidad emocional, sujeto tranquilo, sosegado, relajado y capaz de enfrentarse a situaciones estresantes sin alterarse ni aturdirse), 2) Extraversión (puntuación alta: persona extrovertida, sociable, asertiva, activa, habladora, le gusta la excitación y la estimulación, carácter alegre, animosa, enérgica y optimista; puntuación baja: persona introvertida, reservada, independiente, constante, que prefiere estar solo (no hay que confundirlo con timidez), no se siente desdichado ni pesimista y no sufre necesariamente ansiedad social), 3) Apertura (puntuación alta: abierto, poco convencional, dado a cuestionar la autoridad y dispuesto a aceptar nuevas ideas éticas, sociales y políticas; puntuación baja: tiende a ser convencional en su comportamiento y de apariencia conservadora; prefiere lo familiar a lo novedoso y sus respuestas emocionales son en cierto modo apagadas), 4) Amabilidad (puntuación alta: persona amable, altruista, simpatiza con los demás, está dispuesta a ayudarles y cree que los otros se sienten igualmente satisfechos de hacer lo mismo; puntuación baja: persona desagradable o antipática, egocéntrica, suspicaz respecto a las intenciones de los demás y más bien opositora que cooperadora), y 5) Responsabilidad (puntuación alta: persona responsable, voluntariosa, decidida, con voluntad de lograr objetivos, suele tener buen rendimiento académico-profesional, escrupulosa, puntual y fiable; puntuación baja: persona poco rigurosa en aplicar principios morales porque es descuidada en luchar por sus objetivos, puede tender a una pulcritud compulsiva o a una tendencia de conducta de adicción al trabajo). El test consta de 60 afirmaciones, y el sujeto debe informar su grado de acuerdo con su contenido. EI NEO-FFI se desarrolló como una forma abreviada del NEO-PI. La selección de elementos se llevó a cabo utilizando factores validimex (McCrae y Costa, 1989), teniendo como criterio el NEO-PI. Se partió de una muestra de 983 varones y mujeres a quienes se les aplicó el NEO-PI en 1985 (McCrae y Costa, 1988), se factorializaron los 188 ítems y se 
extrajeron los cinco principales componentes, y se utilizó el validimex de rotación factorial de los ítems con el fin de maximizar la validez convergente y discriminante con los factores validimex del NEO-PI.

\section{Resultados}

Síntomas psicopatológicos y dimensiones de personalidad en adolescentes con DV: diferencias entre sexos

En primer lugar, después de realizar la prueba de Levene para verificar el supuesto de homocedastidad y habiendo encontrado igualdad de varianzas, se calcularon las medias, desviaciones típicas y, se realizaron análisis de varianza (ANOVA) con la finalidad de explorar si existen diferencias entre ambos sexos en síntomas psicopatológicos y rasgos de personalidad, cuyos resultados se presentan en la Tabla 1. Los resultados del análisis de varianza multivariante (MANOVA) realizado para el conjunto de los síntomas psicopatológicos puso de relieve la existencia de diferencias significativas entre ambos sexos, Traza de Pillai, $F(12$, 16) $=3,32, p=0,014$, siendo el tamaño del efecto grande $\left(\eta^{2}=0,714, r=0,84\right)$. Los ANOVA (ver Tabla 1) muestran diferencias estadísticamente significativas entre chicos y chicas en varios síntomas psicopatológicos. En muchos síntomas (obsesión-compulsión, depresión, ansiedad, hostilidad, adicionales), en el índice sintomático general, y en el total de síntomas positivos, las chicas con DV tienen puntuaciones superiores a los chicos con DV.

Por otro lado, los resultados del análisis de varianza multivariante (MANOVA) realizado para las 5 dimensiones de personalidad mos- tró que no existen diferencias significativas entre ambos sexos, Traza de Pillai, $F(5,23)=$ $2,47, p=0,062$, siendo el tamaño del efecto medio $\left(\eta^{2}=0,350, r=0,59\right)$. Los resultados de los análisis univariantes (ver Tabla 1) confirmaron que no existen diferencias en ninguna de las dimensiones exploradas, es decir, que chicos y chicas con DV tuvieron similares niveles en las 5 dimensiones de personalidad evaluadas.

Con la finalidad de explorar si existen diferencias en la psicopatología y en la personalidad entre chicos y chicas adolescentes con y sin discapacidad, se compararon las puntuaciones obtenidas en este estudio realizado con adolescentes con DV, con las puntuaciones de los baremos de las muestras de tipificación de los tests elaborados con adolescentes con visión normal.

En primer lugar, se comparan los resultados obtenidos en esta muestra de adolescentes con DV con los resultados obtenidos en un estudio previo (Garaigordobil et al., 2008) en el que se elaboraron baremos para el SCL-90-R con 236 adolescentes de la CAPV (115 chicos y 121 chicas) de las mismas edades pero con visión normal. La comparación de los resultados permite observar que los chicos con DV, comparados con los chicos sin discapacidad, obtienen puntuaciones inferiores al centil 75; es decir, las puntuaciones de los chicos con o sin discapacidad son muy similares, y además las puntuaciones de los adolescentes con discapacidad no superan el centil 75 del baremo en ninguna variable del SCL-90. Por consiguiente, los chicos con DV no tienen puntuaciones altas en sín- 
Tabla 1

Diferencias de sexo en síntomas psicopatológicos y dimensiones de personalidad en adolescentes con discapacidad visual

\begin{tabular}{|c|c|c|c|c|c|}
\hline \multirow[b]{2}{*}{ Síntomas Psicopatológicos } & \multicolumn{2}{|c|}{$\begin{array}{c}\text { Chicos } \\
(n=16)\end{array}$} & \multicolumn{2}{|c|}{$\begin{array}{l}\text { Chicas } \\
(n=13)\end{array}$} & \multirow{2}{*}{$\begin{array}{c}\text { Anova } \\
F(1,27)\end{array}$} \\
\hline & $M$ & $D T$ & $M$ & $D T$ & \\
\hline Somatización & 0,59 & 0,53 & 0,98 & 0,61 & 3,28 \\
\hline Obsesión-compulsión & 0,81 & 0,67 & 1,59 & 0,86 & $6,19 *$ \\
\hline Sensibilidad interpersonal & 0,73 & 0,62 & 1,18 & 0,78 & 2,98 \\
\hline Depresión & 0,63 & 0,47 & 1,24 & 0,85 & $5,96 *$ \\
\hline Ansiedad & 0,50 & 0,51 & 1,00 & 0,79 & $4,28 *$ \\
\hline Hostilidad & 0,57 & 0,55 & 1,14 & 0,79 & $5,14 *$ \\
\hline Ansiedad fóbica & 0,36 & 0,38 & 0,35 & 0,47 & 0,00 \\
\hline Ideación paranoide & 1,00 & 0,65 & 1,10 & 0,87 & 0,13 \\
\hline Psicoticismo & 0,39 & 0,43 & 0,65 & 0,77 & 1,29 \\
\hline Adicionales (depresión melancólica) & 0,70 & 0,58 & 1,38 & 0,71 & $7,87^{* *}$ \\
\hline GSI Índice sintomático general & 0,62 & 0,38 & 1,07 & 0,65 & $5,18 *$ \\
\hline PST Total de síntomas positivos & 34,31 & 17,55 & 50,15 & 15,39 & $6,50 *$ \\
\hline PSDI Índice distrés síntomas positivos & 1,62 & 0,45 & 1,83 & 0,62 & 1,16 \\
\hline \multicolumn{6}{|l|}{ Dimensiones Personalidad } \\
\hline Neuroticismo & 19,50 & 6,18 & 23,77 & 6,11 & 3,45 \\
\hline Extraversión & 30,75 & 5,88 & 31,31 & 6,25 & 0,06 \\
\hline Apertura & 25,56 & 5,87 & 23,77 & 4,76 & 0,78 \\
\hline Amabilidad & 32,19 & 5,87 & 29,23 & 6,28 & 1,70 \\
\hline Responsabilidad & 26,69 & 8,08 & 27,23 & 4,81 & 0,02 \\
\hline
\end{tabular}

${ }^{*} p<0,05 * * p<0,01$

tomas psicopatológicos mostrando puntuaciones similares a los chicos que no tienen discapacidad. Sin embargo, las puntuaciones de las chicas con DV al interpretarlas con los baremos realizados con chicas adolescentes sin discapacidad, corresponden a centiles de 85 o superiores en muchos síntomas (obsesión-compulsión, ansiedad, hostilidad, ideación paranoide, psicoticismo, adicionales), así como en el índice sintomático general (GSI) y en el índice de distrés por síntomas positivos (PSDI). Así, las chicas con DV tienen puntuaciones altas en muchos síntomas psicopatológicos y en el índice global de psicopatología, y también tienen puntuaciones diferencialmente más altas que las chicas sin discapacidad de estas edades.
Por otro lado, al comparar las puntuaciones obtenidas por los adolescentes con DV de este estudio con las obtenidas en la muestra de tipificación del test presentada en el manual de la prueba y elaborada con adolescentes con visión normal se puede observar que comparados con éstos, las puntuaciones en neuroticismo en chicos y chicas están dentro de la media (centil 50 y 40 respectivamente), el nivel de extraversión es algo bajo (centil 25 en ambos sexos), la apertura en los chicos está dentro de la media (centil 45), sin embargo, en las chicas es bastante baja (centil 10), en amabilidad los chicos tienen puntuaciones altas (centil 85) y las chicas puntuaciones medias (centil 45), y en responsabilidad su nivel está dentro de la media (centil 40 y 45 respectivamente). Por lo 
tanto, los chicos adolescentes con DV comparados con los adolescentes sin discapacidad muestran niveles algo bajos de extraversión, y bastante altos de amabilidad; mientras que las chicas tienen niveles algo bajos de extraversión y muy bajos de apertura.

Síntomas psicopatológicos y dimensiones de personalidad: cambios en función de la edad

En tercer lugar, para analizar los cambios evolutivos que se producen en ambas variables se llevaron a cabo análisis descriptivos y de varianza cuyos resultados se exponen en la Tabla 2. Los resultados del análisis de varianza multivariante (MANOVA) realizado para el conjunto de los síntomas psicopatológicos puso de relieve que en síntomas psicopatológicos no existen diferencias significativas entre los 4 rangos de edad evaluados, Traza de Pillai, $F(36$, 48) $=1,08, p=0,388$, siendo el tamaño del efecto grande $\left(\eta^{2}=0,449, r=0,67\right)$. Los ANOVA (ver Tabla 2) muestran que no existen diferencias evolutivas en la mayoría de los síntomas evaluados, excepto en los síntomas de hostilidad. Los resultados del análisis de comparación de medias de Tukey confirman que en síntomas de hostilidad existen diferencias entre los rangos de edad 14-15 y 16-17, con una puntuación superior en el rango 16-17. Por lo tanto, únicamente se observan diferencias significativas en los síntomas de hostilidad con una puntuación superior a los 16-17 años, no obstante, los datos evidencian que comparadas las puntuaciones entre los 4 rangos de edad, a los 16-17 años se observan las puntuaciones más altas en diversos síntomas (obsesión-compulsión, depresión, ansiedad, ideación paranoide, adi- cionales) así como en el índice sintomático general, aunque las diferencias entre niveles de edad no son estadísticamente significativas.

Por otro lado, los resultados del análisis de varianza multivariante (MANOVA) realizado para las 5 dimensiones de personalidad analizadas globalmente evidenciaron la existencia de diferencias significativas en función de la edad, Traza de Pillai, $F(15,69)=1,98, p=0,029$, siendo el tamaño del efecto grande $\left(\eta^{2}=0,302\right.$, $r=0,54$ ). No obstante, los ANOVA (ver Tabla 2) muestran diferencias estadísticamente significativas únicamente en la dimensión responsabilidad. Los resultados de la prueba de Tukey permiten observar diferencias significativas entre los rangos de edad 12-13 y 18-20, con una puntuación significativamente superior en responsabilidad en el rango de 12-13 años. Así, únicamente se han encontrado diferencias estadísticamente significativas en responsabilidad con un nivel superior en el menor nivel de edad (12-13 años), observándose una disminución de la responsabilidad a medida que aumenta la edad.

En cuarto lugar, con la finalidad de valorar el papel mediador del sexo en los cambios evolutivos, se analiza la interacción entre edad y sexo en síntomas psicopatológicos y dimensiones de personalidad para lo que se llevan a cabo análisis descriptivos (medias y desviaciones típicas) y de varianza cuyos resultados se muestran en la Tabla 3. Los resultados del análisis multivariante (MANOVA) realizado para el conjunto de los síntomas psicopatológicos puso de relieve que la interacción edad $x$ sexo en síntomas psicopatológicos no fue significativa, Traza de Pillai, $F(36,36)=0,95, p$ 
Tabla 2

Diferencias en función de la edad en síntomas psicopatológicos y dimensiones de personalidad en adolescentes con discapacidad visual

\begin{tabular}{cccc}
\hline $12-13$ años & $14-15$ años & $16-17$ años & $18-20$ años \\
$(n=5)$ & $(n=10)$ & $(n=8)$ & $(n=6)$
\end{tabular}

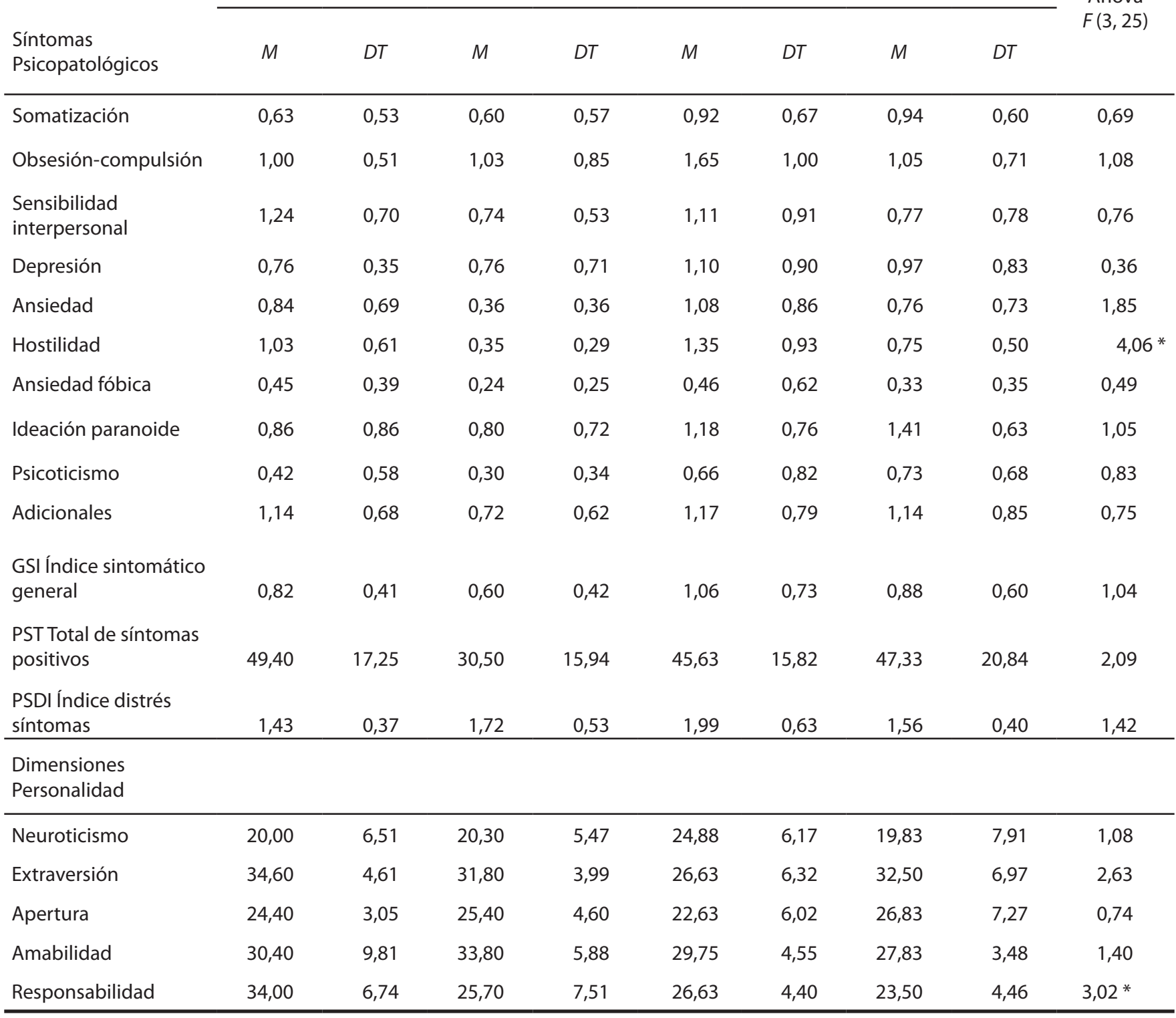

${ }^{*} p<.05$

$=0,550$, siendo el tamaño del efecto grande $\left(\eta^{2}=0,489, r=0,69\right)$. En la misma dirección apuntan los resultados del análisis multivariante realizado para las dimensiones de personalidad, confirmando que la interacción edad $x$ sexo no fue significativa, Traza de PiIlai, $F(15,57)=0,34, p=0,986$, siendo el tama- ño del efecto pequeño $\left(\eta^{2}=0,084, r=0,28\right)$. Tal y como se puede observar en la Tabla 3, los resultados de los análisis de varianza evidenciaron que la interacción entre edad y sexo no fue significativa en ninguno de los síntomas psicopatológicos ni en las 5 dimensiones de personalidad. Por consiguiente, el sexo no 
se confirma como mediador de los cambios evolutivos en las variables evaluadas. No obstante, tal y como se pone de relieve en los distintos síntomas psicopatológicos, en los rangos 16-17 años y 18-20 años, las mayores elevaciones que se dan en estos niveles de edad se deben especialmente a las superiores puntuaciones de las chicas.

Relaciones entre síntomas psicopatológicos y dimensiones de personalidad

Con la finalidad de analizar las relaciones existentes entre psicopatología y personalidad se calcularon los coeficientes de correlación de Pearson entre las puntuaciones del SCL-90-R y del NEO-FFI, cuyos resultados se exponen en la Tabla 4. Los coeficientes obtenidos confirman relaciones significativas positivas en ambos sexos entre neuroticismo y muchos síntomas psicopatológicos (obsesión-compulsión, sensibilidad interpersonal, depresión, ansiedad, ansiedad fóbica, ideación paranoide, adicionales), así como con el índice sintomático general (GSI), y con el índice de distrés por síntomas positivos (PSDI). Además, se encontraron correlaciones positivas, pero únicamente en la muestra de chicos, del neuroticismo con síntomas (hostilidad, psicoticismo), y con el índice total de síntomas positivos (PST). En segundo lugar, se encontró que extraversión tuvo correlaciones significativas negativas con síntomas de obsesión-compulsión en ambos sexos. También, se hallaron correlaciones negativas pero únicamente en la muestra de chicos entre extraversión y síntomas de depresión, y de ansiedad fóbica, mientras que en la muestra de chicas se dieron correlaciones negativas con síntomas de sensibilidad interpersonal, ansiedad, ideación paranoide, psicoticismo, y con los índices GSI (índice sintomático general) y PSDI (índice de distrés por síntomas positivos). En relación al rasgo apertura se encontraron correlaciones positivas con síntomas adicionales pero sólo en la muestra de chicos. En la dimensión amabilidad se hallaron correlaciones negativas con síntomas adicionales pero sólo en la muestra de chicos. En la dimensión responsabilidad se encontraron correlaciones negativas con síntomas de depresión, e ideación paranoide sólo en la muestra de chicos. Las dimensiones apertura, amabilidad y responsabilidad, en la muestra de chicas, no correlacionaron con ningún síntoma ni indicador de psicopatología.

\section{Discusión}

En primer lugar, los resultados obtenidos confirman que las chicas con DV, en comparación con los chicos con DV, tienen puntuaciones significativamente superiores en varios síntomas psicopatológicos (obsesión-compulsión, depresión, ansiedad, hostilidad, adicionales), en el índice sintomático general, y en el total de síntomas positivos, sin embargo, no existen diferencias entre ambos sexos en ningún rasgo de personalidad. Estos datos confirman la hipótesis 1 en su totalidad, ya que las chicas muestran más síntomas psicopatológicos, y no hay diferencias entre sexos en las dimensiones de la personalidad. Estos resultados apuntan en la misma dirección que los hallados por Dimitriou (1973) que observó mayor frecuencia de síntomas en las chicas adolescentes que estudiaban en una escuela para ciegos, y tam- 


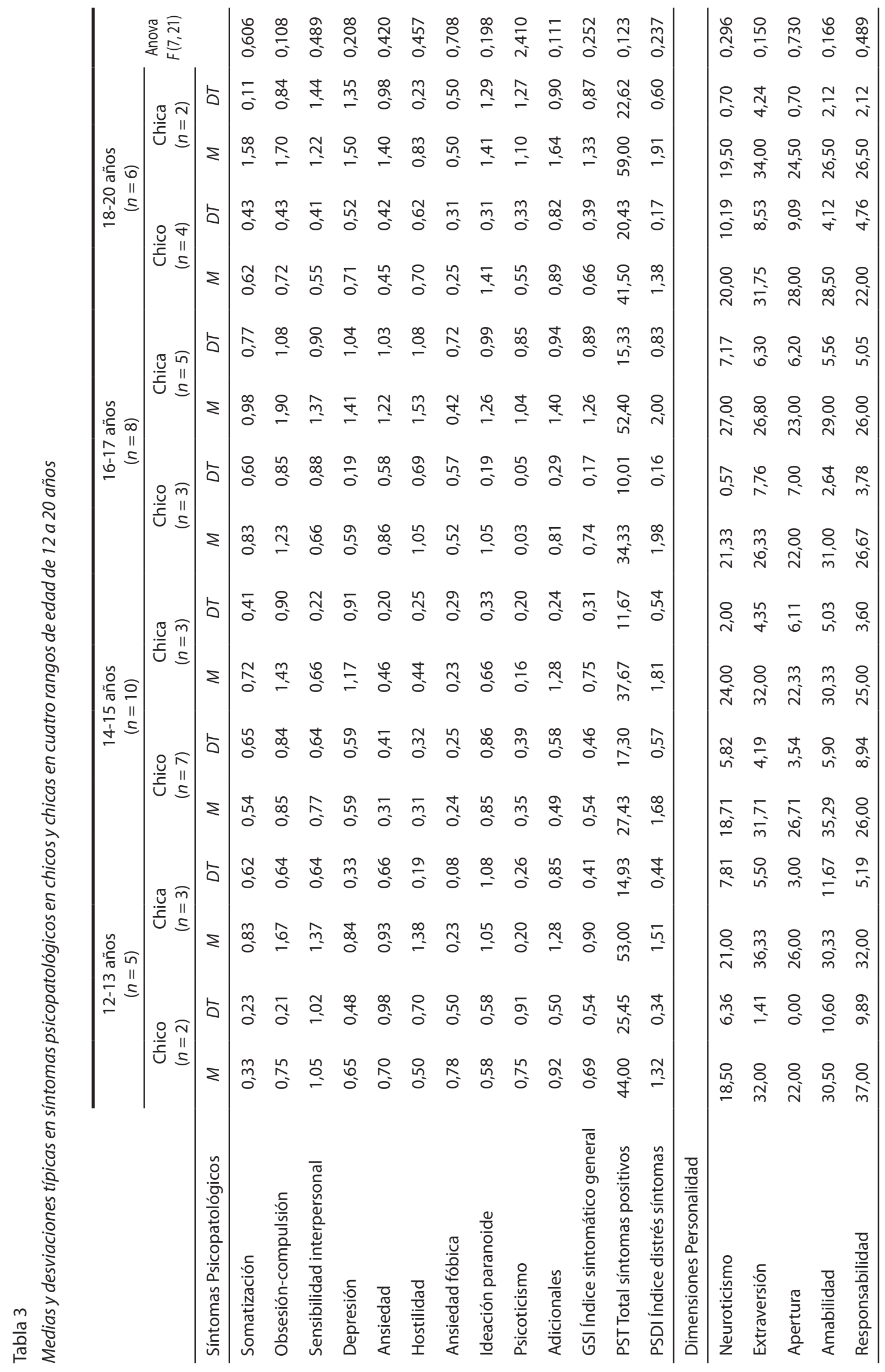


bién confirman los obtenidos en el estudio de Garaigordobil et al. (2008) en el que encontraron un nivel mayor de síntomas psicopatológicos en las mujeres de visión normal frente a los hombres en todas las edades (12 a 65 años). dimensiones de personalidad, chicos y chicas con DV (frente a los de sin discapacidad) muestran niveles bajos de extraversión y además las chicas tienen niveles muy bajos de apertura. Por lo tanto, la hipótesis 2 se confirma parcial-

Tabla 4

Correlaciones de Pearson entre síntomas psicopatológicos y dimensiones de personalidad para chicos $(n=16)$ y chicas $(n=13)$ de forma independiente

\begin{tabular}{lcccccccccc} 
& \multicolumn{2}{c}{ Neuroticismo } & \multicolumn{2}{c}{ Extraversión } & \multicolumn{2}{c}{ Apertura } & \multicolumn{2}{c}{ Amabilidad } & \multicolumn{2}{c}{ Responsabilidad } \\
\cline { 2 - 10 } & Chico & Chica & Chico & Chica & Chico & Chica & Chico & Chica & Chico & Chica \\
\cline { 2 - 10 } Somatización & 0,45 & 0,43 & $-0,10$ & $-0,37$ & 0,29 & 0,21 & $-0,03$ & $-0,36$ & $-0,27$ & 0,10 \\
Obsesión- & & & & & & & & & & \\
compulsión & $0,69^{* *}$ & $0,76^{* *}$ & $-0,52^{*}$ & $-0,72^{* *}$ & 0,40 & 0,10 & $-0,08$ & $-0,15$ & $-0,42$ & $-0,01$ \\
Sensibilidad & & & & & & & & & & \\
interpersonal & $0,54^{*}$ & $0,60^{* *}$ & $-0,21$ & $-0,55^{*}$ & 0,42 & 0,11 & $-0,05$ & $-0,14$ & $-0,26$ & 0,12 \\
Depresión & $0,90^{* * *}$ & $0,58^{*}$ & $-0,43^{*}$ & $-0,51$ & 0,44 & 0,08 & $-0,08$ & $-0,07$ & $-0,55^{*}$ & $-0,09$ \\
Ansiedad & $0,56^{*}$ & $0,67^{*}$ & $-0,12$ & $-0,63^{*}$ & 0,01 & 0,07 & $-0,43$ & $-0,30$ & $-0,24$ & 0,16 \\
Hostilidad & $0,68^{* *}$ & 0,52 & $-0,33$ & $-0,37$ & 0,03 & 0,09 & $-0,39$ & $-0,00$ & $-0,30$ & 0,23 \\
Ansiedad fóbica & $0,53^{* *}$ & $0,59^{*}$ & $-0,65^{* *}$ & $-0,49$ & 0,08 & 0,16 & $-0,40$ & $-0,09$ & $-0,04$ & 0,10 \\
Ideación paranoide & $0,65^{* *}$ & $0,63^{*}$ & $-0,20$ & $-0,62^{*}$ & 0,23 & 0,30 & $-0,12$ & $-0,37$ & $-0,55^{*}$ & 0,07 \\
Psicoticismo & $0,50^{*}$ & 0,50 & $-0,00$ & $-0,63^{*}$ & 0,16 & 0,12 & $-0,17$ & $-0,11$ & $-0,41$ & $-0,02$ \\
Adicional & $0,65^{* *}$ & $0,63^{*}$ & $-0,34$ & $-0,54$ & $0,54^{*}$ & 0,48 & $-0,54^{*}$ & $-0,23$ & $-0,24$ & 0,01 \\
GSI & $0,87^{* * *}$ & $0,69^{* *}$ & $-0,39$ & $-0,64^{*}$ & 0,39 & 0,13 & $-0,23$ & $-0,18$ & $-0,47$ & 0,05 \\
PST & $0,78^{* * *}$ & 0,40 & $-0,38$ & $-0,48$ & 0,42 & 0,38 & $-0,45$ & $-0,41$ & $-0,38$ & 0,22 \\
PSDI & $0,51^{*}$ & $0,75^{* *}$ & $-0,17$ & $-0,59^{*}$ & 0,16 & 0,06 & 0,24 & 0,00 & $-0,26$ & $-0,13$ \\
\hline
\end{tabular}

${ }^{*} p<0,05{ }^{* *} p<0,01{ }^{* * *} p<0,001$

En segundo lugar, los resultados muestran que las chicas con DV, comparadas con las de visión normal, tienen puntuaciones altas (percentiles superiores a 85) en diversos síntomas psicopatológicos (obsesión-compulsión, ansiedad, hostilidad, ideación paranoide, psicoticismo, adicionales), en el índice global de psicopatología y en número total de síntomas positivos. Sin embargo, los chicos con DV tienen puntuaciones medias y similares a los adolescentes sin discapacidad. En relación a mente, ya que se había propuesto que los y las adolescentes con DV tendrían puntuaciones superiores en el índice global de psicopatología y en el rasgo de personalidad neuroticismo. Los resultados de este estudio contradicen los hallazgos del trabajo de Koenes y Karshmer (2000) que encontró un nivel significativamente mayor de depresión en 22 adolescentes ciegos, pero podría ser explicado por el hecho de que los adolescentes de su muestra eran ciegos de nacimiento. Y también los estudios 
realizados con adultos de 60 o más años en los que también se han evidenciado síntomas de depresión (Chou y Chi, 2004; Noran et al., 2009) que en gran medida podrían ser explicadas por la edad de estas muestras. No obstante, nuestros resultados son coherentes con los obtenidos en otros trabajos que no han hallado puntuaciones superiores en depresión en los adolescentes con discapacidad (frente a los de sin discapacidad) (Garaigordobil y Bernarás, 2009). Los resultados del estudio aportan información relevante en relación a los síntomas psicopatológicos en los adolescentes con DV y evidencian las importantes diferencias entre sexos, ya que mientras que las chicas con discapacidad tienen más psicopatología que las chicas sin discapacidad, esto no se da en los chicos con DV que tienen niveles de psicopatología similares a los chicos sin discapacidad. Por otro lado, en relación al bajo nivel de extraversión y de apertura, tal vez la falta de visión no facilita las relaciones sociales con las personas, normalmente los adolescentes con DV tienen tendencia a escuchar, más que a intervenir, no ven a quién tienen delante y eso dificulta la apertura y la extraversión, prefieren pasar desapercibidos, no llamar la atención, prefieren, como dicen en el propio test lo familiar a lo novedoso, con respuestas emocionales apagadas.

En tercer lugar, los resultados de los análisis de varianza muestran que no existen diferencias entre los 4 rangos de edad en la mayoría de los síntomas psicopatológicos evaluados, excepto en los síntomas de hostilidad, en los que se observan puntuaciones superiores a los 16-17 años. En las dimensiones de perso- nalidad únicamente se ha observado una disminución de la responsabilidad a medida que aumenta la edad. Los resultados han evidenciado que la interacción edad x sexo en síntomas psicopatológicos y en personalidad no fue significativa, por lo que el sexo no emerge como un mediador en los cambios evolutivos. Estos resultados no confirman la hipótesis 3 que proponía que a medida que aumentara la edad iba a haber un incremento de los síntomas psicopatológicos y del neuroticismo, que en el resto de las dimensiones de personalidad no iba a haber variaciones con la edad, y que el sexo iba a mediar los cambios evolutivos con mayor nivel de cambio en las chicas. En relación a la disminución de la responsabilidad con la edad que se ha observado tal vez pueda ser explicada por la sobreprotección que en muchos casos tienen estos adolescentes y que no les ayuda a luchar por sus objetivos, a ser decididos. Son aspectos que deberían tener en cuenta los padres y educadores. En este sentido, se podría proponer que tengan contacto con personas más mayores que han vivido esa situación y que con valentía han conseguido los objetivos que se han propuesto en la vida.

Finalmente, los resultados de los análisis correlacionales han evidenciado que los chicos y chicas adolescentes con DV que tenían altas puntuaciones en neuroticismo también tenían muchos síntomas psicopatológicos (de obsesión-compulsión, sensibilidad interpersonal, depresión, ansiedad, ansiedad fóbica, ideación paranoide, adicionales), así como un nivel alto de sintomatología general y de malestar por los síntomas. Y los resultados también han confirmado que los y 
las adolescentes con altas puntuaciones en extraversión tenían pocos síntomas de obsesión-compulsión. Por consiguiente la hipótesis 4 se confirma parcialmente ya que sí se han encontrado relaciones positivas entre síntomas psicopatológicos y neuroticismo, también se han hallado relaciones positivas entre extraversión y síntomas de obsesióncompulsión en ambos sexos, y con otros síntomas psicopatológicos aunque mayormente en la muestra de chicas, sin embargo, no se han encontrado relaciones negativas entre psicopatología y dimensiones como amabilidad y responsabilidad. Estos datos apoyan la importancia de educar en habilidades sociales y resaltar modelos y programas que puedan darles confianza en sí mismos a los y las adolescentes con DV.

Algunos estudios (Oduntan, 2005) han subrayado que la DV a menudo se asocia a manifestaciones psicológicas como el estrés, depresión, negación, ansiedad, pobre autoconcepto... Y desde estos trabajos han planteado que la rehabilitación del paciente con DV debe incluir una intervención psicológica. Aunque la contribución de la psicoterapia a la rehabilitación de los pacientes con DV ha sido reconocida durante varias décadas, los servicios de los psicólogos son raramente empleados por los optometristas y oftalmólogos, que son los que por lo general están a cargo de los pacientes que requieren rehabilitación visual. Una posible razón de esto es que habitualmente se ha subestimado la importancia de la intervención psicológica en la rehabilitación visual. En relación a esta cuestión y teniendo en cuenta la variabilidad de los resultados obtenidos en los estudios, así como los resultados de nuestro trabajo con adolescentes se puede sugerir, como ya ha sido señalado por otros investigadores (Ammerman et al., 1986; Freedman, 1967; Norris et al., 1957), que la DV no es en sí misma necesariamente una causa de mal ajuste o de psicopatología y que existe un número importante de variables intervinientes, tanto relacionadas con la gravedad de la discapacidad como con factores ambientales. Por consiguiente, y dada la variabilidad en torno a síntomas psicopatológicos y otras variables relacionadas con el bienestar psicológico en las personas con DV (factores etiológicos, gravedad de la discapacidad, ambiente familiar, edad ) será importante estudiar cada caso individualmente, de cara a recomendar intervención psicológica así como sobre las características de la misma.

Los resultados permiten sugerir el desarrollo de intervenciones para disminuir los síntomas psicopatológicos en las chicas adolescentes con DV, para fomentar la extraversión en los y las adolescentes con DV.Y también programas de fomento de la autoestima, de habilidades sociales, que planteen retos de menor a mayor escala, que les ayude a ser menos hostiles, confiar más en los semejantes, ser más responsables y con voluntad de lograr los objetivos que se propongan. Como limitación del estudio se puede destacar el bajo número de participantes, aunque hay que tener en cuenta que la población de sujetos de edades comprendidas entre 12 y 20 años con DV y sin ninguna otra discapacidad asociada en la CAPV es pequeña. Entre las líneas futuras de investigación se puede plantear evaluar cómo elaboran sus 
proyectos de vida futuros los adolescentes con

DV y si existen diferencias de género en esa elaboración.

\section{Bibliografía}

Ammerman, R. T., Van Hasselt, V. B., \& Hersen, M. (1986). Psychological adjustment of visually handicapped children and youth. Clinical Psychology Review, 6, 67-85.

Burlingham, D. (1979). To be blind in a sighted workd. The Psychoanalytic Study of the Child, 34, 5-30.

Casten, R. J., Rovner, B. W., \& Edmonds, S. E (2001). The relationships among personality and vision-specific function among older people with impaired vision. Journal of Mental Health and Aging, 7, 325-334.

Chou, K. L., \& Chi, I. (2004). Combined effect of vision and hearing impairment on depression in elderly Chinese. International Journal of Geriatric Psychiatry, 19, 825-832.

Organización Mundial de la Salud (OMS) (1992). CIE-10. Décima Revisión de la Clasificación Internacional de las Enfermedades. Trastornos mentales y del comportamiento. Madrid: Meditor.

Costa, P., \& McCrae, R. (1999). Inventario de personalidad NEO revisado (NEO PI-R). Inventario NEO reducido de cinco factores (NEO-FFI). Madrid:TEA.

De las Cuevas, C., \& González de Rivera, J. L. (1991). Perfil sintomático y diagnóstico en pacientes psiquiátricos ambulatorios. Psiquis, 12, 326-336.

Derogatis, L. R., Rickels, K., \& Rock, A.F. (1976). The SCL-90 and the MMPI: A step in the validation of a new self-report scale. British Journal of Psychiatry, 128, 280-289.

Derogatis, L. R., \& Cleary, P. A. (1977). Confirmation of the dimensional structure of the SCL-90: A study in construct validation. Journal of Clinical Psychology, 33, 981-989.
Derogatis, L. R. (1983). SCL-90-R, Administration, scoring and procedures Manual II for the revised version of the SCL-90. Baltimore: Johns Hopkins University Press.

Dimitriou, E. C. (1973). Neuroticism in blind children, adolescents and young adults. Research Bulletin. American Foundation for the Blind, 26, 261.

Fiesler, W., Lechner, L., \& Bos, A. (2008). If you can't see--see if I care... An experimental study on the effects of seriousness, personal responsibility and openness on stigmatization of visually impaired persons. [Als je niet kunt zien dan bekijk je het maar...Experimenteel onderzoek naarde effecten van ernst, persoonlijke verantwoordelijkheid en openheid op stigmatise ring van mensen met een visuele beperking]. Psychologie \& Gezondheid, 36, 23-31.

Freedman, S. (1967). Psychological implications of the multiply handicapped person. New Outlook for the Blind, 61, 185-189.

Garaigordobil, M., \& Bernarás, E. (2009). Selfconcept, self-esteem, personality traits and psychopathological symptoms in adolescents with and without visual impairment. Spanish Journal of Psychology, 12, 149-160.

Garaigordobil, M., Pérez, J. I., \& Mozaz, M. (2008). Self-concept, self-esteem and psychopathological symptoms. Psicothema, 20, 114-123.

González de Rivera, J. L., De las Cuevas, C., Rodríguez Abuín, M. V., \& Rodríguez Pulido, F. (2002). SCL-90-R, Symptom Cheklist 90 Revised, Adaptación española. Madrid: TEA.

Huurre, T., \& Aro, H. (1998). Psychosocial development among adolescents with visual impairment. European Child \& Adolescent Psychiatry, 7, 73-78.

Huurre, T., \& Aro, H. (2000). The psychosocial wellbeing of Finnish adolescents with visual impairments versus those with chronic conditions and those with no disabilities. Journal of Visual Impairment \& Blindness, 94, 625-637. 
Koenes, S. G., \& Karshmer, J. F. (2000). Depression: A comparison study between blind and sighted adolescents. Issues in Mental Health Nursing, 21, 269-279.

McCrae, R., \& Costa, P. (1988). From catalog to classification: Murray's needs and five-factor model. Journal of Personality and Social Psychology, 55, 258-265.

McCrae, R., \& Costa, P. (1989). Rotation to maximize the construct validity of factors in the NEO pertsonatity inventory. Multivariate Behavioral Research, 24, 107-124.

Noran, N. H., Izzuna, M. G., Bulgiba, A. M., Mimiwati, Z., \& Ayu, S. M. (2009). Severity of visual impairment and depression among elderly Malaysians. Asia-Pacific Journal of Public Health, 21, 43-50.

Norris, M., Spaulding, P. J., \& Brodie, F. H. (1957). Blindness in children. Chicago: University of Chicago Press.

Oduntan, A. O. (2005). The role of psychotherapy in the contemporary rehabilitation of visually impaired patients. En Ntomchukwu Sylvester Madu y Saraswathie Govender (Eds.), Mental health and psychotherapy in Africa (pp. 308-320). Sovenga, South Africa: UL Press of the University of Limpopo-Turfloop Campus.

Rovner, B. W., \& Casten, R. J. (2001). Neuroticism predicts depression and disability in age-related macular degeneration. Journal of the American Geriatrics Society, 49, 10971100.

Satapathy, S., \& Singhal, S. (2001). Predicting social-emotional adjustment of the sensory impaired adolescents. Journal of Personality Clinical Studies, 17, 85-93. 